\title{
Limits and Confidence Intervals in the Presence of Nuisance Parameters
}

\author{
Wolfgang A. Rolke ${ }^{\mathrm{a}}$, Angel M. López ${ }^{\mathrm{b}}$, Jan Conrad ${ }^{\mathrm{c}}$ \\ ${ }^{a}$ Department of Mathematics, University of Puerto Rico - Mayagüez, Mayagüez, PR 00681, \\ $U S A$, \\ Postal Address: PO Box 5959, Mayagüez, PR 00681, \\ Tel: (787) 255-1793, Email: wolfgang@puerto-rico.net \\ ${ }^{b}$ Department of Physics, University of Puerto Rico - Mayagüez, Mayagüez, PR 00681, USA \\ ${ }^{c}$ PH-Department, CERN, CH-1211, Geneva 23, Switzerland.
}

\begin{abstract}
We study the frequentist properties of confidence intervals computed by the method known to statisticians as the Profile Likelihood. It is seen that the coverage of these intervals is surprisingly good over a wide range of possible parameter values for important classes of problems, in particular whenever there are additional nuisance parameters with statistical or systematic errors. Programs are available for calculating these intervals.
\end{abstract}

Key words: Maximum likelihood, coverage, Monte Carlo, sensitivity

Preprint submitted to Elsevier

February 14, 2014 


\section{Introduction}

The calculation of confidence intervals (or setting of limits) on a parameter of a theory is one of the most important problems an experimental physicist can face. In the frequentist approach which we follow here, the main property which confidence intervals have to fulfill is to have coverage. A method is said to yield a $100(1-\alpha) \%$ confidence interval if, were the experiment to be repeated many times, the resulting intervals would include (or cover) the true parameter at least $100(1-\alpha) \%$ of the time, no matter what the true parameter is. Using a construction method due to Neyman [1], Feldman and Cousins [2] in 1998 found confidence intervals for the case of one nuisance parameter when its value is known exactly. An alternative method used widely in high energy physics prior to the publication of Feldman and Cousins is to extract confidence intervals by finding the points where the -2log likelihood function increases by a factor defined by the required confidence level ( $\ln \mathcal{L}+\frac{1}{2}$ method). This method is also well known in Statistics as the large-sample approximation to the likelihood ratio test statistic. One major drawback of this method is that, because it is based on a large-sample theory, its performance for small samples is in question. In fact, it is known that the $\ln \mathcal{L}+\frac{1}{2}$ method has under-coverage in certain circumstances. However, it does not suffer from the few practical drawbacks of Feldman and Cousins. Firstly, it can be generalized to the case where there are many parameters of interest. Secondly (and more importantly here) it is easily adapted to treat problems with several nuisance parameters which are not known exactly.

In this note we will combine the $\ln \mathcal{L}+\frac{1}{2}$ method with what is known in Statistics 
as the profile likelihood method in which the multi-dimensional likelihood function is reduced to a function that only depends on the parameter of prime interest. The combination of the profile likelihood approach with the $\ln \mathcal{L}+\frac{1}{2}$ extraction of confidence intervals is well known to particle physicists from the program MINUIT [3], 4] and to astrophysicists from SERROR [5] and is discussed at http://www.sr.bham.ac.uk/asterix-docs/Programmer/Source/Algorithms/serror.html.

For the problem of setting confidence limits for the signal rate in the presence of background which is estimated from data sidebands or Monte Carlo, this approach has previously been shown in Rolke and López [6] to have good coverage. In this note the method will be generalized to the problem of a signal with a Poisson distribution, a background with either a Poisson or a Gaussian distribution and an efficiency with either a Binomial or a Gaussian distribution. We will establish the domain of validity, enabling comparison with other methods. As we will show, this method, together with some minor adjustments, has very good coverage even in cases when the parameters lie close or at the physical boundaries.

Although this paper, and the corresponding routines, only deal with the specific problems outlined above, the results show that the method of profile likelihood is a viable technique for dealing with nuisance parameters, and it should be useful for other problems as well. 


\section{The Method}

\subsection{Profile Likelihood}

The basic idea of the profile likelihood is rather straightforward: assume we have a probability model for our data which depends on parameters $\boldsymbol{\pi}=$ $\left(\pi_{1}, . ., \pi_{k}\right)$ of interest to the researcher but also on additional nuisance parameters $\boldsymbol{\theta}=\left(\theta_{1}, . ., \theta_{l}\right)$. If we denote the probability mass function (or density) by $f(x \mid \boldsymbol{\pi}, \boldsymbol{\theta})$ and we have independent observations $\mathbf{X}=\left(X_{1}, . ., X_{n}\right)$, then the full likelihood function is given by

$$
L(\boldsymbol{\pi}, \boldsymbol{\theta} \mid X)=\prod_{i=1}^{n} f\left(X_{i} \mid \boldsymbol{\pi}, \boldsymbol{\theta}\right)
$$

If, as is often done in Statistics, we decide to base our inference on the likelihood, our challenge is to eliminate the nuisance parameters.

A standard technique for constructing confidence intervals is to find a corresponding hypothesis test, and then to invert that test. Here the hypothesis test is $H_{0}: \boldsymbol{\pi}=\boldsymbol{\pi}_{0}$ vs $H_{a}: \boldsymbol{\pi} \neq \boldsymbol{\pi}_{0}$ and a test can be based on the likelihood ratio test statistic given by

$$
\lambda\left(\boldsymbol{\pi}_{0} \mid X\right)=\frac{\sup \left\{L\left(\boldsymbol{\pi}_{0}, \boldsymbol{\theta} \mid X\right) ; \boldsymbol{\theta}\right\}}{\sup \{L(\boldsymbol{\pi}, \boldsymbol{\theta} \mid X) ; \boldsymbol{\pi}, \boldsymbol{\theta}\}}
$$

The supremum in the denominator is found over the full parameter space, whereas the supremum in the numerator is found only over the subspace with $\boldsymbol{\pi}=\boldsymbol{\pi}_{0}$. Notice that $\lambda$ is a function of $\boldsymbol{\pi}_{0}$ (and the data) only, but does not depend on the nuisance parameters $\boldsymbol{\theta}$. In the context of nuisance parameters the function $\lambda$ is also called the profile likelihood. One of the standard results from 
Statistics (see for example Casella and Berger [7]) is that $-2 \log \lambda$ converges in distribution to a chi-square random variable with $k$ degrees of freedom. This in fact is the theoretical basis for the $\ln \mathcal{L}+\frac{1}{2}$ method for extracting limits from the likelihood function.

We illustrate the method of profile likelihood using the example of a search for a rare decay where the expected background is known only approximately. We will need the following notation. Assume that we observe $x$ events in a suitably chosen signal region and a total of $y$ events in the background region. Here the background region can be chosen fairly freely and need not be contiguous. Furthermore, the probability that a background event falls into the background region divided by the probability that it falls into the signal region is denoted by $\tau$. For example, if we use two background regions of the same size as the signal region and assume the background distribution is flat we have $\tau=2$. If the background rate is estimated from Monte Carlo, $\tau$ is the size of the Monte Carlo sample relative to the size of the data sample. Then a probability model for the data is given by

$$
X \sim \operatorname{Pois}(\mu+b), \quad Y \sim \operatorname{Pois}(\tau b)
$$

where $\mu$ is the signal rate, $b$ is the background rate and Pois is the usual Poisson distribution. We will use large caps letters $X, Y$ to denote random variables and small caps letters $x, y$ to denote realizations (observed values) of these random variables. We can assume $X$ and $Y$ to be independent and so

$$
f(x, y \mid \mu, b)=\frac{(\mu+b)^{x}}{x !} e^{-(\mu+b)} \cdot \frac{(\tau b)^{y}}{y !} e^{-\tau b}
$$


The likelihood function is given by $L(\mu, b \mid x, y)=f(x, y \mid \mu, b)$. Maximizing over both $\mu$ and $b$ we find the usual maximum likelihood estimators $(\widehat{\mu}, \widehat{b})=$ $(x-y / \tau, y / \tau)$. Fixing $\mu$ and maximizing over $\mathrm{b}$ alone yields

$$
\hat{b}(\mu)=\frac{x+y-(1+\tau) \mu+\sqrt{(x+y-(1+\tau) \mu)^{2}+4(1+\tau) y \mu}}{2(1+\tau)}
$$

For other models it may not be possible to find $\hat{b}(\mu)$ analytically, in which case numerical methods need to be used. Now the profile likelihood function is given by

$$
\lambda(\mu \mid x, y)=\frac{L(\mu, \hat{b}(\mu) \mid x, y)}{L(\widehat{\mu}, \widehat{b} \mid x, y)}
$$

and according to the theory $-2 \log \lambda$ has an approximate $\chi^{2}$ distribution with 1 degree of freedom.

For more details on the likelihood ratio test statistic see Casella and Berger [7]. For information on the profile likelihood see Bartlett [8], Lawley [9] and Murphy and Van Der Vaart [10].

For the studies conducted here, the maximizations leading to $-2 \log \lambda$ have mostly been done analytically, but we have also verified that the program MINUIT (which does this numerically) produces the same results when using MINOS errors if $x \geq y / \tau$. The case of $x<y / \tau$ is discussed in detail in section 2.3 .

There are a number of refinements known in Statistics for the profile likelihood. We had to make a judgement call here: keep the method very simple and easy to implement for different types of problems, or include further refinements which might improve the method. Given that it works as well as it does any 
improvement would be very slight, at least for the problems studied in detail. We consider it a definite strength of our method that it should be very easy to apply to other problems, and this advantage would be lost if we advocated such refinements.

\subsection{The Treatment of Efficiency and Systematic Errors}

The general nature of the profile likelihood technique for dealing with nuisance parameters can be illustrated by considering several modifications and extensions of the problem as laid out in the previous paragraphs. For example, say we want to include the efficiency $e$ into our limits. Assume that we are Monte Carlo limited and therefore have to deal with the error in the efficiency

estimate. Specifically, say we run $m$ events through our Monte Carlo (without background) and find $z$ events surviving. Then we can model the efficiency $Z$ as a binomial random variable and find the complete model to be

$$
X \sim \operatorname{Pois}(e \mu+b), \quad Y \sim \operatorname{Pois}(\tau b), \quad Z \sim \operatorname{Bin}(m, e)
$$

where Bin is the binomial distribution. To find the profile likelihood we fix $\mu$ and differentiate the loglikelihood:

$$
\begin{gathered}
\frac{\partial}{\partial b} \log l(\mu, b, e \mid x, y, z)=\frac{x}{e \mu+b}-1+\frac{y}{b}-\tau \doteq 0 \\
\frac{\partial}{\partial e} \log l(\mu, b, e \mid x, y, z)=\frac{x}{e \mu+b}-\mu+\frac{z}{e}-\frac{m-z}{1-e} \doteq 0
\end{gathered}
$$

This system of nonlinear equations can not be solved analytically but we can do so numerically, and again we have the profile likelihood curve as a function of the signal rate $\mu$ alone. 
As a second example, suppose that the background and the efficiency are better modeled as Gaussians rather than using the Poisson and the Binomial, for example, to allow the inclusion of systematic errors. Then we have the probability model

$$
X \sim \operatorname{Pois}(e \mu+b), \quad Y \sim N\left(b, \sigma_{b}\right), \quad Z \sim N\left(e, \sigma_{e}\right)
$$

where $N$ indicates the Gaussian (or normal) distribution and $\sigma_{b}$ and $\sigma_{e}$ are the standard deviations or errors on the estimates of $b$ and $e$, respectively. Now we find the derivatives of the loglikelihood to be

$$
\begin{aligned}
& \frac{\partial}{\partial b} \log l(\mu, b, e \mid x, y, z)=\frac{x}{e \mu+b}-1+\frac{(y-b)}{\sigma_{b}} \doteq 0 \\
& \frac{\partial}{\partial e} \log l(\mu, b, e \mid x, y, z)=\frac{x}{e \mu+b}-\mu+\frac{(z-e)}{\sigma_{e}} \doteq 0
\end{aligned}
$$

This system can actually be solved analytically.

All combinations of the above models, such as the background modeled as a Poisson and the efficiency modeled as a Gaussian as well as the cases where one or the other or both are known without error, are equally easily treated.

\subsection{Extracting Limits}

As was discussed above $-2 \log \lambda$ has approximately a chi-square distribution, and this can be used to extract limits. Other methods also exist, for example, using information from the second derivative of the log profile likelihood. However, for the specific problem of rare decays, this does not yield a method with correct coverage. 
Figure 1 shows $-2 \log \lambda$ for the case $x=8, y=15, \tau=5.0$. To find a $100(1-\alpha) \%$ confidence interval we start at the minimum, which of course is at the usual maximum likelihood estimator, and then move to the left and to the right to find the points where the function increases by the $\alpha$ percentile of a $\chi^{2}$ distribution with 1 degree of freedom. For example, if we want to find a $90 \%$ confidence interval, the increase will be 2.706 .

Some care must be taken in the cases where the maximum likelihood estimator is small or negative. If it is positive but sufficiently small, $-2 \log \lambda$ might not increase enough to the left within the physical region $(\mu \geq 0)$. In that case, we set the lower limit to zero. (All our techniques produce physically valid limits.)

In the cases where fewer events are observed in the signal region than are expected from background, the log profile likelihood curve, just like the regular log likelihood, is no longer parabolic. Rolke and López [6] dealt with this problem by using a hypothesis test based on the null hypothesis $H_{0}: \mu=\mu_{0}, b=b_{0}$, deriving the corresponding two-dimensional acceptance region and then finding the values of $\mu$ where the profile likelihood $(\mu, \hat{b}(\mu))$ enters and leaves the acceptance region. Rolke and López [6] also extended this method to a problem with two nuisance parameters but this becomes computationally very demanding because it requires searching for boundary points of a highly irregular object in two (or more) dimensions.

In this paper we consider two methods for treating these cases. In the first, referred to as the unbounded likelihood method, we proceed just as described above. This is possible because for the problems studied here we can always 
find the maximum likelihood estimators analytically and therefore the value of $-2 \log \lambda$ at that point. The maximum likelihood estimator for the signal rate is negative but we set the lower limit to zero. As an example consider the left panel of figure 2 . Here we have $x=2, y=15, \tau=5.0$, so the maximum likelihood estimator of the signal rate is $\widehat{\mu}=-1.0$. Using the method of unbounded likelihood we find a $95 \%$ upper limit of 3.35 .

In the most extreme case when we observe many fewer events than are expected from background alone, the profile likelihood curve at $\mu=0$ might already be higher than the increase from the minimum. Rather than quoting a negative (and non-physical) upper limit we will find the upper limit by increasing the value of $x$ by 1 until we find the first positive upper limit. This will yield a legitimate upper limit because our limits are monotonically increasing in $x$.

A special treatment is also necessary for the cases $x=0$ and/or $y=0$. This is due to the fact that here the loglikelihood function is linear and therefore does not have a minimum, that is, the maximum likelihood estimator does not exist. We solve this problem by computing the "neighboring" limits $(x=1,2$ and/or $y=1,2)$ and doing a linear extrapolation. This is a reasonable "adjustment" because the limits are nearly linear in $x$ and $y$, and as we shall see in the next section the resulting confidence intervals have good coverage properties.

A second possible solution to the problem of fewer events in the signal region than are expected from background is to use the physical limits on the parameters. So instead of using the increase from the maximum likelihood estimator $\widehat{\mu}$ we instead use the increase from the point $\mu=0$ if $\widehat{\mu}<0$. This is equivalent 
to finding the minimum using MINUIT with a lower bound for the signal rate of 0 . We will refer to this as the method of bounded likelihood. It is illustrated in the right panel of figure 2. Using this method we find a $95 \%$ upper limit of 3.6.

The adjustment for the cases $x=0$ and/or $y=0$ described above is also necessary for the bounded likelihood method because without it the method does not have coverage; for example, a nominal $90 \%$ confidence interval has a true coverage of less than $68 \%$ for some parameter values.

The adjustments described here are rather ad-hoc, and their only theoretical justification is that they make the limits larger, therefore can not make the coverage worse. Their practical justification is of course the fact that the resulting limits have coverage. Such ad-hoc adjustments are not at all unusual in Statistics. Certainly one would prefer a method that deals uniformly with all possible values in data space, but such methods are actually very rare.

The treatment here deals only with the statistical aspect of the problem of having fewer events in the signal region than are expected from background. In such a situation we recommend to also quote the experimental sensitivity which is defined as the mean upper limit for an ensemble of experiments with the observed background and efficiency levels but no signal. In the case where there are fewer events in the signal region than expected from background, the experimental sensitivity gives a better idea of the experimental precision than the upper limit. An even better way to proceed in such cases is to use a "bootstrap procedure", described in Rolke and Lopez [1]], to reduce the effects 
of statistical fluctuations in the background. However, such considerations are outside the scope of this paper. Sensitivity determinations and the bootstrap procedure require what we provide here, a method for calculating limits given any set of observations.

Finally, the method does not return limits for some cases where the Gaussian model for the efficiency is suspect, that is, cases where $z / \sigma_{e}$ is small. One example is shown in figure 3 where we have $x=5, y=2.5, \sigma_{b}=0.4$ and $z=0.2$. Then for $\sigma_{e}=0.1$ the curve rises sufficiently so we can quote an upper limit (85.9) but if $\sigma_{e}=0.15$ it does not. It is impossible to give a precise value for the ratio below which no limits are returned since it also depends on the values of the observations and the confidence level desired. In practice, if the method returns limits, coverage studies (discussed in Section 3) show them to be reliable. If it does not, alternative models for the efficiency distribution will need to be considered.

\section{Performance of this Method}

In the case of confidence intervals, performance means first of all "coverage", that is, a nominal $90 \%$ confidence interval should cover the true value of the parameter at least $90 \%$ of the time for all parameter values. Because in the specific problem discussed here at least one of the observations has a discrete distribution, it is not possible to achieve the nominal coverage for all parameter values. Some overcoverage is unavoidable, although less overcoverage is of course preferable and is a measure of the quality of the method. 
Coverage studies for the case of a Poisson model for the signal and a Poisson model for the background have previously been published in Rolke and López [6]. For the case of an added efficiency modeled as a Binomial (discussed above), consider the following coverage study: We have $\tau=3.5$, the efficiency is $e=0.85$ and $m=100$. We vary the signal rate $\mu$ from 0 to 10 in steps of 0.1 and the background rate $b$ from 0 to 10 in steps of 2 . For each of these 600 combinations of the parameters we find the true coverage of nominal $90 \%$ confidence intervals based on 10000 Monte Carlo runs and for each of the methods described above. The results are shown in figure 4. As a second example we model both the background and the efficiency as Gaussians, with $\sigma_{b}=0.5$ and $\sigma_{e}=0.075$. Again we have $e=0.85$ and vary $\mu$ and $b$ as above. The results are shown in figure 5 .

In both figure 4 and figure 5 we included the case $b=0$ to show that our method is capable of dealing even with such an extreme case with values at the boundary of parameter space.

The coverage of the method is quite good, with only some very small acceptable undercoverage due to the fact that we are using a large-sample approximation. We have some overcoverage for small $\mu$ because the upper limit can not be too small in this case but this is unavoidable. Finally we see that these coverage graphs are considerably smoother with much less overcoverage than those shown in Rolke and López [6] for both the unified method by Feldman and Cousins as well as the method described there. This is because of the higher randomness due to the extra random variable $Z$ for the efficiency. The overcoverage of the 
unbounded likelihood method is generally a little smaller than for the bounded likelihood method. This is as expected because in the cases where they differ the unbounded likelihood method yields lower upper limits than the bounded likelihood method. Extensive coverage studies of over 25000 parameter combinations and for all the models discussed above have shown these results to be quite general.

In our coverage studies we do not include simulation runs for which our method does not return limits. To have "coverage" is to make sure a fixed (and known) percentage of published intervals actually contain the true parameter value. The cases where no limit is returned will not lead to incorrect intervals but rather to a reevaluation of the efficiency model. Such cases should occur very rarely in practice and, in fact, this is so for the coverage studies in figures 4 and 5. A coverage study where the percentage of such cases is substantial is presented in table 1 . Here we have $\mu=5, b=2.5, \sigma_{b}=0.4, \sigma_{e}=0.1$ and we find $90 \%$ confidence intervals. The method has coverage for all values of the parameters, even the extreme value where $e / \sigma_{e}=1$. Although it does overcover under these circumstances, it does give conservative limits. Even for values of $e / \sigma_{e}$ as small as 3 where the percentage of cases that do not return a limit is $8 \%$, the degree of overcoverage is small. However, the Gaussian model should be reconsidered for such small ratios.

Table 1: Percentage of simulation runs that do not return limits and coverage for cases where $e / \sigma_{e}$ is small: 


\begin{tabular}{|r|r|r|}
\hline$e\left(e / \sigma_{e}\right)$ & No Limits & Coverage \\
\hline \hline $0.5(5.0)$ & $0.04 \%$ & $90 \%$ \\
\hline $0.4(4.0)$ & $0.8 \%$ & $90 \%$ \\
\hline $0.3(3.0)$ & $8 \%$ & $92 \%$ \\
\hline $0.2(2.0)$ & $35 \%$ & $96 \%$ \\
\hline $0.1(1.0)$ & $73 \%$ & $98 \%$ \\
\hline
\end{tabular}

In figure 6 we show the behavior of the limits as functions of the uncertainties in background (left panel) and efficiency (right panel). The limits are for the case $x=5, y=3, z=0.5$, and we model both the background and the efficiency as Gaussians. In the left panel we vary the uncertainty in the background rate from 0.0 to 1.0 with the uncertainty in the efficiency fixed at 0.1 . In the right panel we vary the uncertainty in the efficiency from 0.0 to 0.15 with the uncertainty in the background fixed at 0.75 . As we can see the behavior of the limits here is what one expects: the larger the uncertainty the higher the limit. We also see that the limits found using this method are self-consistent, that is, as the uncertainty becomes small the limits smoothly approach a limiting value.

As the coverage graphs show, the unbounded likelihood method generally has less overcoverage than the bounded likelihood method. This is due to the fact that when they differ the upper limits of the unbounded likelihood method are always smaller. This can be seen as an advantage and a reason to prefer the unbounded likelihood method to the bounded likelihood method.

Based on our coverage studies we recommend to use the presented method for Poisson statistics when the uncertainties in nuisance parameters can be modeled 
as one of the seven cases we studied here. For problems other than these the profile likelihood method should be useful as well, but then additional coverage studies need to be carried out.

\section{Comparison with other methods}

An alternative to the method described in this paper is based on integrating over the nuisance parameters, see for example Cousins and Highland [12] and Conrad et. al. [13]. There the full confidence interval construction is performed, but the probability density function is obtained from folding the primary PDF with the one describing the distribution of the nuisance parameters. Treating nuisance parameters in this way is based on Bayesian statistics with a flat prior. The coverage of this method has been studied in Tegenfeldt and Conrad [14]. It appears that treating nuisance parameters in a Bayesian way generally introduces a modest amount of overcoverage, though it has to be emphasized that this has been shown only for the assumed flat prior distribution. In general so-called credible intervals found via Bayesian statistics do not have the property of coverage, and the frequentist properties of such intervals will have to be studied for each problem and each prior anew.

Feldman [15] suggested to use the profile likelihood in the ratio ordering of the unified approach in an effort to extend that method to the case of background uncertainty. Coverage studies for this method have not been presented yet.

A standard method for finding errors, and therefore limits, is based on the second derivative of the log likelihood function. For the problem described here, 
though, this method can have a true coverage of less than $50 \%$ for a nominal $90 \%$ confidence interval, and is therefore not a viable option.

As discussed above our method is very similar to MINUIT/MINOS; in fact for the case $x>y / \tau$ the two yield identical limits. MINUIT/MINOS alone, though, does not have coverage because it does not include the adjustments described in section 2.3.

In figure 6 we have included the limits from Feldman and Cousins unified approach in the case of zero uncertainty in the background rate. Those limits are slightly different from ours. That different methods yield different limits is very common in Statistics because the requirement of coverage is in fact a rather general one and does not uniquely determine the limits.

\section{Implementation}

A stand-alone FORTRAN routine for calculating these limits is available at http://charma.uprm.edu/ rolke/publications.htm. It is also available as TRolke which is part of ROOT [16]. Both routines also calculate the experimental sensitivity described above. Finally, at least for the cases $x>y / \tau$, one could use MINUIT/MINOS to carry out the calculations, though in this case care needs to be taken to set the limits on the parameters correctly.

It is to be hoped that the profile likelihood method yields good results also in situations other than the ones discussed here. Because it is already available as part of MINUIT, its implementation for different problems should be quite straightforward. It needs to be emphasized, though, that the profile likelihood 
method can not be assumed to yield good results in all cases and that it might require some adjustments to the general method as we have done here. It is therefore strongly recommended that a thorough check of its performance be done whenever it is applied to a new problem. In the case of setting limits, this means a coverage study as described above, at least for the range of likely parameter values.

\section{Summary}

We have discussed the method of profile likelihood as a general treatment of nuisance parameters within a frequentist framework. For the case of a Poisson distributed signal with a background that has either a Poisson or a Gaussian distribution and an efficiency that has either a Binomial or a Gaussian distribution, we have carried out an extensive coverage study and shown that the method yields confidence intervals with good coverage throughout the parameter space, even at its boundaries.

\section{Acknowledgements}

J.C. would like to thank R. Brun for support with ROOT and G. Stefanini for his patience. All authors would like to thank F. James for pointing out the connection to MINUIT and for various discussions that have allowed us to improve this paper considerably. This work was supported by the Division of High Energy Physics (Grant DE-FG-97ER41045) of the US Department of Energy and the University of Puerto Rico. 


\section{References}

[1] J.Neyman, "Outline of a Theory of Statistical Estimation based on the Classical Theory of Probability", Phil. Trans. Royal Soc. London, A236 (1937) 333.

[2] R.D. Cousins, G.J. Feldman, "A Unified Approach to the Classical Statistical Analysis of Small Signals", Phys. Rev, D57, (1998) 3873.

[3] F. James, M. Roos, "'MINUIT', a System for Function Minimization and Analysis of the Parameter Errors and Correlations", Comput. Phys. Commun.10 (1975) 343-367

[4] F. James, "Interpretation of the Shape of the Likelihood Function around its Minimum", Comput. Phys. Commun,.20, (1980) 29-35

[5] M. Lampton, B. Margon and S. Bowyer, "Parameter Estimation in X-ray Astronomy", Astrophysical Journal. 208, (1976) p177-190

[6] W.A. Rolke, A.M. López, "Confidence Intervals and Upper Bounds for Small Signals in the Presence of Background Noise", Nucl. Inst. and Methods A458 (2001) 745-758.

[7] G. Casella, R.L. Berger, Statistical Inference, Duxburry Press, (1990) 346.

[8] M.S. Bartlett, "Approximate Confidence Intervals, Part II: More than one Unknown Parameter", Biometrica Vol. 40, (1953) 306-317.

[9] D.N. Lawley, "A General Method For Approximating To The Distribution Of The Likelihood Ratio Criteria", Biometrica Vol. 43, (1956) 295-303. 
[10] S.A. Murphy, A.W. Van Der Vaart, "On Profile Likelihood", Journal of the American Statistical Association, Vol. 95, (2000), 449-485.

[11] W.A. Rolke, A.M. López, "Correcting the Minimization Bias in Searches for Small Signals", Nucl. Inst. and Methods A503/3, 2003, 617 - 624

[12] R. D. Cousins and V.L. Highland, "Incorporating Systematic Uncertainties into an Upper Limit", Nucl. Inst. and Methods A320 (1992) 331-335.

[13] J. Conrad, O. Botner, O, A. Hallgren, A. and C. Perez de los Heros, "Including Systematic Uncertainties in Confidence Interval Construction for Poisson Statistics", Phys. Rev, D67, (2003) 12002.

[14] F. Tegenfeldt and J.Conrad, "On Bayesian Treatment of Systematic Uncertainties in Confidence Interval Calculations," accepted for publication in Nucl. Inst. and Methods, arXiv:physics/0408039

[15] G.J. Feldman, "Multiple Measurements and Parameters in the Unified Approach", Workshop on Confidence Limits, Fermilab (2000), http://conferences.fnal.gov/cl2k/copies/feldman2.pdf

[16] R. Brun and F. Rademaker, "ROOT - An Object Oriented Data Analysis Framework", Proceedings AIHENP'96 Workshop, Lausanne, Sep. 1996, Nucl. Inst. \& Meth. in Phys. Res. A 389 (1997) 81-86. See also http://root.cern.ch/

\section{Appendix}




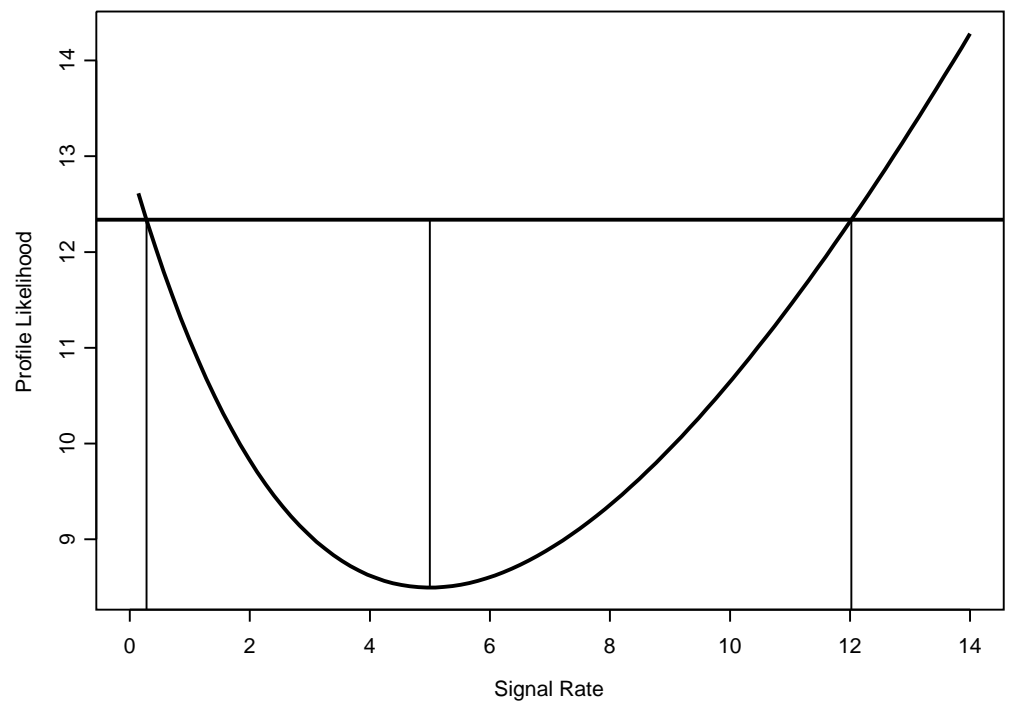

Figure 1: Example of the $-2 \log \lambda$ curve. This is the case $x=8, y=15$ and $\tau=5.0$. We find the $95 \%$ confidence interval to be $(0.28,12.02)$. 

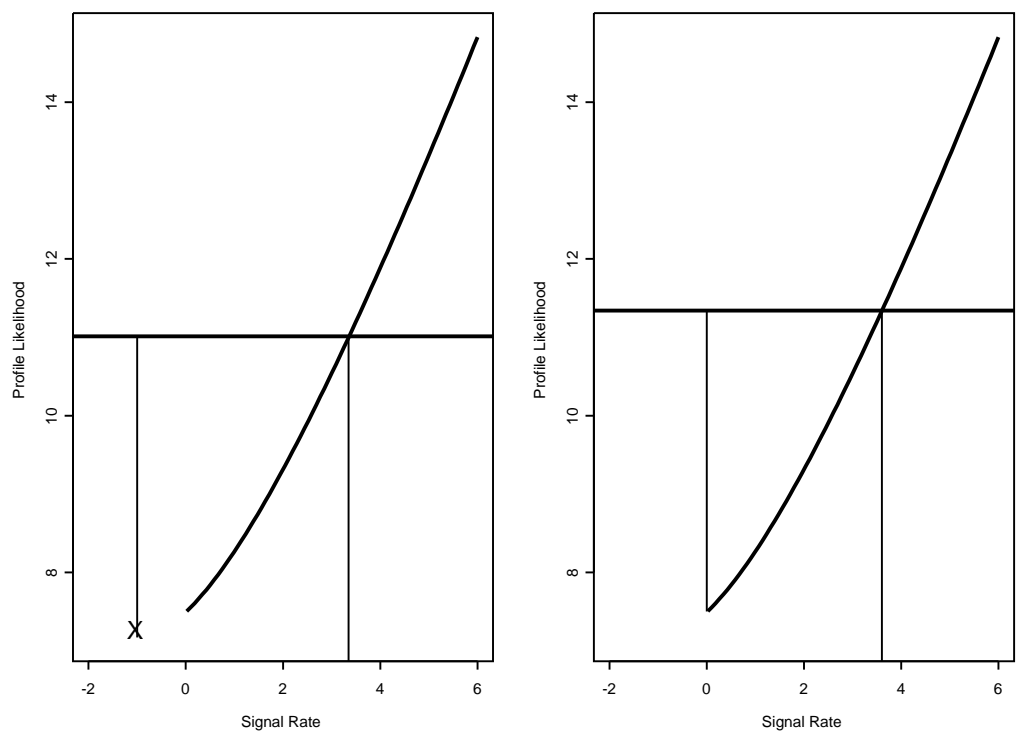

Figure 2: The case $x=2, y=15$ and $\tau=5.0$. In the left panel we use the unbounded likelihood method and find a 95\% upper limit of 3.35. In the right panel using the bounded likelihood method the $95 \%$ upper limit is 3.6 . 


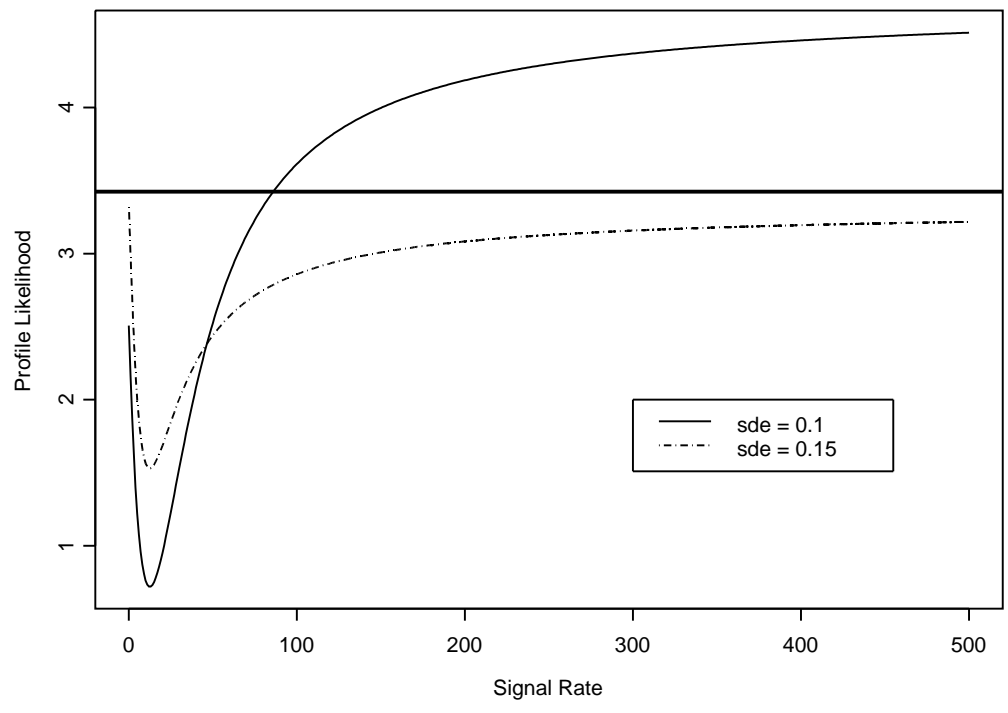

Figure 3: An illustration of why the profile likelihood method does sometimes not return a limit. Here $x=5, y=2.5, \sigma_{b}=0.4$ and $z=0.2$. If $\sigma_{e}=0.1$ the curve moves above the required level ( for a $90 \%$ confidence interval) and the upper limit is 85.9 but if $\sigma_{e}=0.15$ the curve stays below the level and no limit is found. 

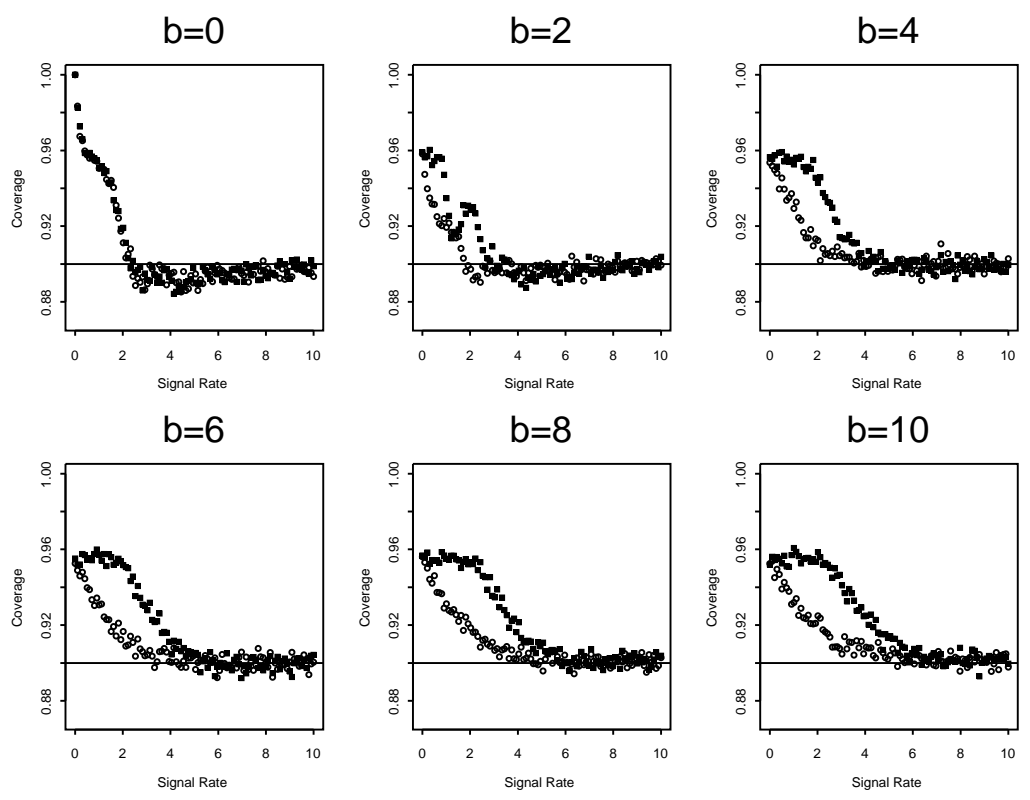

Figure 4: $90 \%$ coverage graphs when the signal and the background are modeled as Poisson and the efficiency is modeled as a Binomial with $\tau=3.5, e=0.85$ and $m=100$. The empty circles show the coverage using the unbounded likelihood method and the solid squares show the coverage using the bounded likelihood method. 

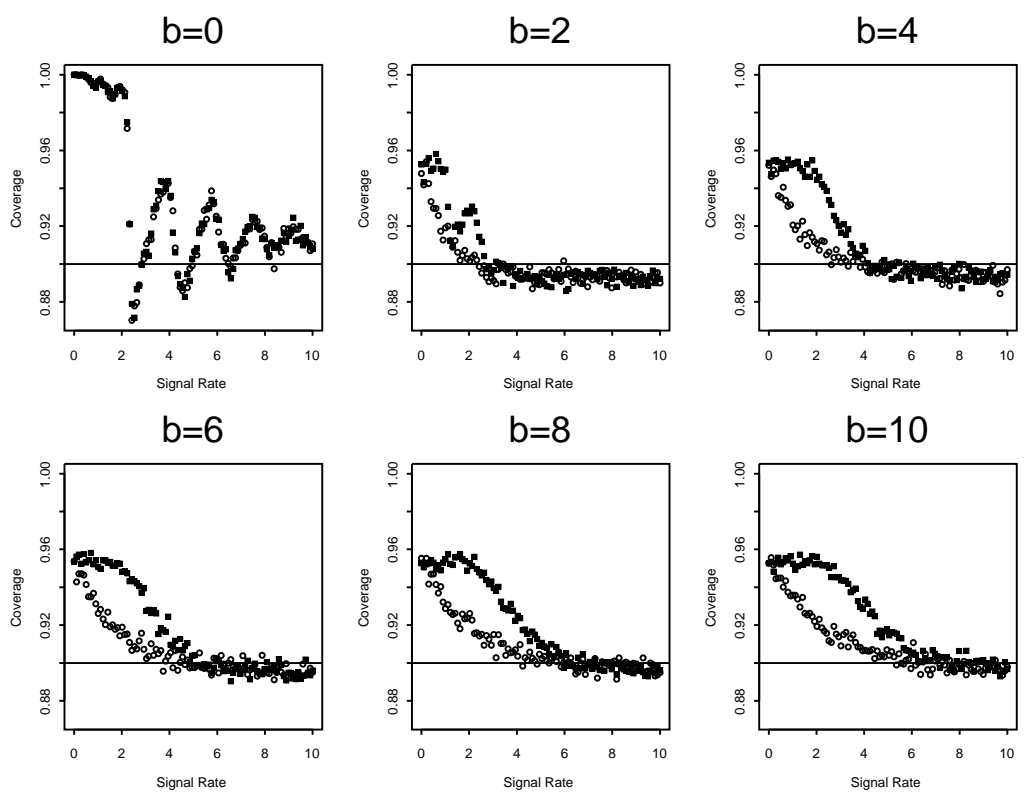

Figure 5: 90\% coverage graphs when the signal is modeled as a Poisson and the background and the efficiency are modeled as Gaussians with $\sigma_{b}=0.5, e=0.85$ and $\sigma_{e}=0.075$. The empty circles show the coverage using the unbounded likelihood method and the solid squares show the coverage using the bounded likelihood method. 


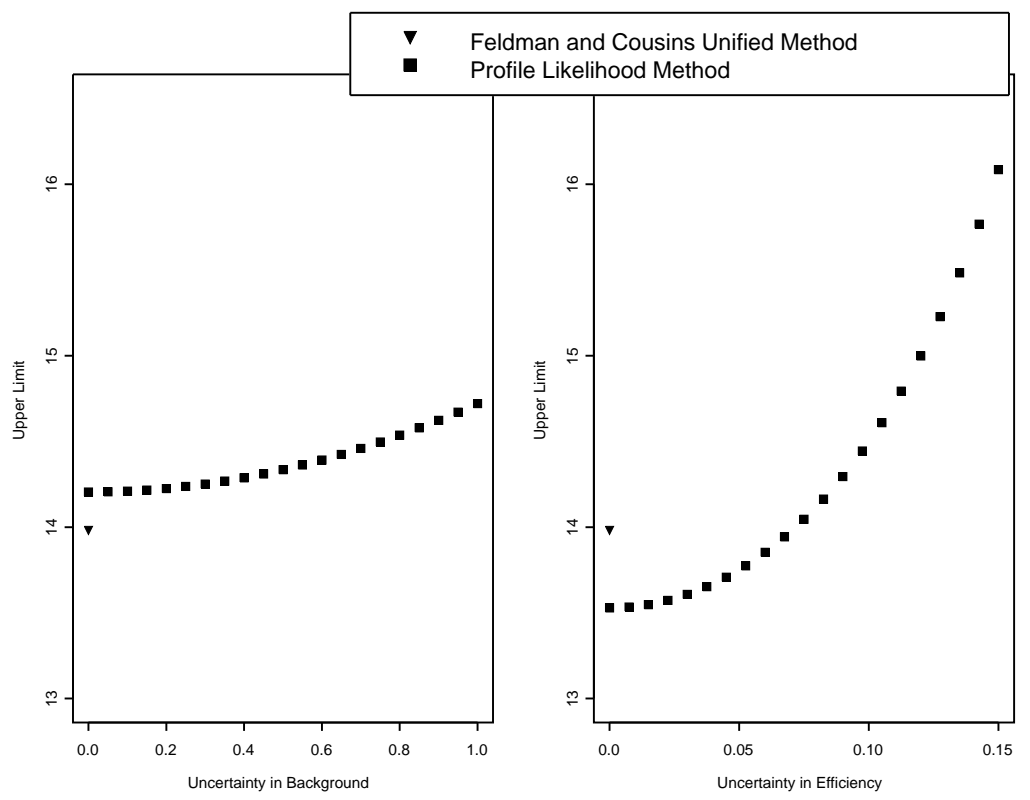

Figure 6: Upper limits as a function of the uncertainties. In both graphs the background and the efficiency are modeled as Gaussians. In the left panel we have the case $x=5, y=3$, $z=0.5, \sigma_{e}=0.1$ and the uncertainty in the background goes from 0.0 to 1.0. In the right panel we have the case $x=5, y=3, z=0.5, \sigma_{b}=0.75$ and the uncertainty in the efficiency goes from 0.0 to 0.15 . We have added the limits derived from Feldman and Cousins unified method which ignores the uncertainties. 